\title{
Prognostic value of IGF-IR expression in bone and soft tissue sarcomas: a meta-analysis
}

This article was published in the following Dove Press journal:

OncoTargets and Therapy

29 July 2015

Number of times this article has been viewed

\section{Junbo Liang ${ }^{1,2}$ \\ Binghao $\mathrm{Li}^{3}$ \\ Li Yuan ${ }^{4}$ \\ Zhaoming $\mathrm{Ye}^{3}$}

'School of Clinical Medicine, Wenzhou Medical University, Wenzhou, ${ }^{2}$ Department of Orthopedics, Taizhou Hospital, Taizhou, ${ }^{3}$ Department of Orthopaedics, Institute of Orthopaedic Research, Second Affiliated Hospital of Zhejiang University School of Medicine, Hangzhou, ${ }^{4}$ School of Public Health, Fudan University, Shanghai, People's Republic of China
Correspondence: Zhaoming Ye Department of Orthopaedics, Institute of Orthopaedic Research, Second Affiliated Hospital, Zhejiang University School of Medicine, Hangzhou, People's Republic of China Email yezhaominghz@163.com

\begin{abstract}
Accumulated evidence has indicated a correlation between IGF-1R and bone and soft tissue sarcoma (BSTS) progression. However, research on the prognostic role of IGF-1R in sarcomas has revealed very different or even totally opposite results. This meta-analysis aimed to unveil the controversial role IGF-1R plays in predicting the outcome of BSTS patients. We systematically reviewed the evidence for the effect of IGF-1R expression in multiple types of BSTSs, including osteosarcoma, Ewing's sarcoma, synovial sarcoma, liposarcoma, and rhabdomyosarcoma, to elucidate this issue. The prognostic value of IGF-1R expression in BSTS patients was evaluated regarding overall survival, measured by pooled hazard ratios (HRs) with 95\% confidence intervals (CIs). Seven studies including 627 patients were enrolled in this metaanalysis. Our results demonstrated that IGF-1R expression was associated with poor outcome in terms of overall survival in BSTS patients (pooled HR $=2.15,95 \% \mathrm{CI}$ : 1.06-4.38; $P=0.03$ ). In subtypes of BSTSs, elevated IGF-1R expression was revealed to be significantly correlated with worse prognosis in osteosarcoma (pooled HR $=2.20,95 \% \mathrm{CI}$ : $1.59-0.03 ; P<0.001$ ), while no statistical significance was discovered in Ewing's sarcoma (pooled HR $=1.01,95 \%$ CI: $0.45-2.27 ; P=0.99$ ). Expression of IGF-1R could be a negative prognostic biomarker for patients suffering from BSTSs.
\end{abstract}

Keywords: overall survival, prognostic significance osteosarcoma, meta-analysis, IGF-1R

\section{Introduction}

Bone and soft tissue sarcomas (BSTSs) are a heterogeneous group of malignant tumors that originate from mesenchymal tissue, including osteosarcoma, Ewing's sarcoma (EWS), synovial sarcoma (SS), liposarcoma, rhabdomyosarcoma, etc. Most of them, especially osteosarcoma and EWS, are aggressive tumors that require complete surgical resection and consequently, affect quality of life. ${ }^{1}$ Although advances in 5-year survival have been achieved in recent decades, patients are still suffering from tumor relapse and metastasis. For patients with low-grade BSTSs, the prognosis is usually not bad, and conventional therapeutic regimens will meet the demand, whereas in highgrade cases, where the prognosis is poor, the puzzling question arises as to whether patients should receive more aggressive treatment. There are still a substantial number of BSTS patients for whom outcomes cannot be reliably predicted during the treatment. Hence, potential prognostic markers, such as some disease-related symptoms and biological indicators, should be evaluated and will consequently help in making therapeutic strategies. In our previous research, we analyzed the prognostic value of pathologic fracture in osteosarcoma. ${ }^{2}$ Nevertheless, a single marker is obviously far from satisfactory in dealing with treatments, and some laboratory examinations should be included. As BSTSs involve a series of tumors, a widely expressed protein may be the optimum choice to analyze its potential in predicting prognosis. 
There is abundant evidence that the insulin-like growth factor (IGF) system plays an important role in the malignancy of BSTSs. ${ }^{3,4}$ IGF pathway-targeted therapy is underway in a substantial number of clinical trials. ${ }^{5,6} \mathrm{How}-$ ever, IGF-1R, a member of the IGF-1 signaling pathway, plays an elusive role in sarcomas. Upregulated expression of IGF-1R was observed in multiple kinds of sarcomas, ${ }^{7}$ and increasing evidences has suggested that IGF-1R is involved in survival of osteosarcoma, ${ }^{8} \mathrm{EWS},{ }^{9} \mathrm{SS},{ }^{10}$ etc. Nevertheless, research on the prognostic role of IGF-1R in BSTSs has revealed very different or even totally opposite results. In osteosarcoma, Wang et $\mathrm{al}^{11}$ demonstrated that patients with elevated IGF-1R expression had poorer survival, and high IGF-1R expression was an independent prognostic maker, whereas a study by van de Luijtgaarden et $\mathrm{al}^{12}$ presented the results that IGF-1R seemed to have a favorable role in overall survival (OVS), although the finding was not statistically significant. It might owe to the interference of IGF-1R pathway which consequently sensitized osteosarcomas to chemotherapy. ${ }^{13,14}$ EWS research by Scotlandi et al ${ }^{15}$ revealed that high levels of IGF-1R expression correlated with favorable outcome, while van de Luijtgaarden et $\mathrm{a}^{16}$ demonstrated that there was no statistical difference in the OVS of patients with or without positive IGF-1R, despite the lower survival rate of patients with highly expressed IGF-1R. In other BSTSs, such as SS, liposarcoma, and rhabdomyosarcoma, IGF-1R was considered to negatively influence survival of patients; $;{ }^{17-19}$ at the same time, a study on multiple soft tissue sarcomas showed a significant association between high expression of IGF-1R and favorable outcome, although the numbers of SS, liposarcoma, and rhabdomyosarcoma were small $\left(\mathrm{n}=3,16\right.$, and 1 , respectively). ${ }^{20}$

In consideration of these conflicting research results, a meta-analysis was needed. In this study, by pooling results from the available data, we sought to make an objective evaluation of the prognostic significance of high IGF-1R expression level for OVS in patients suffering from BSTSs, including osteosarcoma, EWS, SS, liposarcoma, and rhabdomyosarcoma.

\section{Materials and methods Data strategies}

This meta-analysis was performed according to the Preferred Reporting Items for Systematic Reviews and Meta-Analyses (PRISMA) standardized guidelines. A literature search was conducted to identify publications relating to the association of IGF-1R expression with BSTSs. The Web of Science and PubMed databases were searched for the period up to and including April 2015 to locate related publications. The search strategy was based on combinations of the following terms: "IGF-1R", "sarcoma", "osteosarcoma", "Ewing", "synovial", "liposarcoma", "rhabdomyosarcoma". All searches were limited to the English language. Citations that included the key terms in either the title, abstract, article, or Medical Subject Heading (MeSH) terms were retained. The references of retrieved articles also were screened for relevance.

\section{Study selection}

The eligibility of studies was assessed by two independent reviewers (Liang and Li). All contradictions were seriously discussed until a consensus was reached. The abstracts of all candidate articles were read for preliminary screening. Articles that could not be categorized based on the title and abstract were retrieved for a full-text review. These articles were independently read and checked for the inclusion criteria.

\section{Inclusion criteria of studies}

Studies were included according to following criteria: 1) measured IGF-1R expression in the patients with BSTSs by immunohistochemistry (IHC); 2) provided information on overall survival; and 3) had a maximum follow-up time exceeding 5 years. Studies considered ineligible for this meta-analysis were: 1) reviews, editorials, or letters; 2) studies involving patients who underwent IGF-1R targeted therapy; 3) studies that enrolled less than 20 patients in total or five patients per group who underwent molecular analysis, as well as studies for which no hazard ratio (HR) with its 95\% confidence interval (CI) could be calculated from the available data; 4) nonhuman studies; 5) duplicate publications or unqualified data; and 6) studies with NewcastleOttawa Quality Assessment Scale (NOS) score (www.ohri. ca/programs/clinical epidemiology/oxford.htm) for cohort studies of less than 6 .

\section{Data extraction}

Two investigators (Liang and Li) independently extracted data from the eligible studies; they discussed discrepancies and reached consensus for all items. The following information was extracted from each of the included studies: first author's name, year of publication, patient source, number of patients, tumor types, analysis method, HR estimation for OVS, and prognostic value. HR estimates and $95 \%$ CIs were calculated if not provided in the paper directly. The primary 
authors were contacted to obtain the necessary data if the required information was not adequately provided.

\section{Quality assessment}

Quality assessment was conducted for each eligible study using the NOS. The NOS provides relatively objective evaluation criteria for case-control and cohort studies, and the effectiveness and credibility of NOS have been widely approved. A study was considered high-quality if it achieved a score of 7 or more (Table 1).

\section{Statistical analysis}

In this meta-analysis, HR and 95\% CI were used to assess the association of IGF-1R expression with OVS in patients with BSTSs. Some of the included studies provided HR and 95\% CI explicitly. If HR and 95\% CI were not reported, we calculated the values in the original studies or from data provided by author, using the methods illustrated by Tierney et al. ${ }^{21}$ Engauge Digitizer software was used to extract data from the Kaplan-Meier curves, according to the provided protocol. Heterogeneity was assessed using the $\chi^{2}$ test and $I^{2}$ statistics. An $I^{2}$ value more than $50 \%$ was regarded as evidence of high heterogeneity, for which the randomeffects model was utilized to pool the results; otherwise, the fixed-effects model was used to pool the results. Because the characteristics of participants were not consistent between studies, we further conducted subgroup analysis by cancer types (osteosarcoma or EWS), source of patients (Eastern or Western), and sample size (divided by $n=50$ ). Potential publication bias was assessed by visual inspection of the funnel plots, in which the standard error of logHR of each study was plotted against its $\operatorname{logHR}$, and an asymmetric plot suggested possible publication bias. In addition, we also performed Egger's linear regression test, at the $P$-value $<0.10$ level of

Table I Newcastle-Ottawa quality assessment scale

\begin{tabular}{|c|c|c|}
\hline Scale item & Description & Score \\
\hline Selection & $\begin{array}{l}\text { I. Representativeness of the exposed cohort } \\
\text { a. Truly representative of the average sarcoma patients in the community* } \\
\text { b. Somewhat representative of the average sarcoma patients in the community* } \\
\text { c. Selected group of users } \\
\text { d. No description of the derivation of the cohort } \\
\text { 2. Selection of the nonexposed cohort } \\
\text { a. Drawn from the same community as the exposed cohort* } \\
\text { b. Drawn from a different source } \\
\text { c. No description of the derivation of the nonexposed cohort } \\
\text { 3. Ascertainment of exposure (proof of sarcoma and IGF-IR measured by IHC) } \\
\text { a. Secure record (such as surgical records)* } \\
\text { b. Structured interview* } \\
\text { c. Written self-report } \\
\text { d. No description } \\
\text { 4. Demonstration that outcome of interest was not present at start of study } \\
\text { a. Yes* } \\
\text { b. No }\end{array}$ & 4 \\
\hline Comparability & $\begin{array}{l}\text { Comparability of cohorts on the basis of the design or analysis } \\
\text { a. Study controls for IGF-IR expression* } \\
\text { b. Study controls for other factors (age, sex, treatment, etc)* }\end{array}$ & I \\
\hline Outcome & $\begin{array}{l}\text { I. Assessment of outcome (death) } \\
\text { a. Independent blind assessment* } \\
\text { b. Record linkage* } \\
\text { c. Self-report } \\
\text { d. No description } \\
\text { 2. Was follow up long enough for outcomes to occur? } \\
\text { a. Yes ( } 5 \text { years)* } \\
\text { b. No } \\
\text { 3. Adequacy of follow up of cohorts } \\
\text { a. Complete follow up - all subjects accounted for* } \\
\text { b. Subjects lost to follow up unlikely to introduce bias - small number lost }(25 \%) \text { or description } \\
\text { provided of those lost* } \\
\text { c. Follow-up rate }<75 \% \text { and no description of those lost } \\
\text { d. No statement }\end{array}$ & 3 \\
\hline
\end{tabular}

Note: *Indicates significance.

Abbreviation: IHC, immunohistochemistry. 
significance, to assess funnel plot asymmetry. All analyses were performed using RevMan version 5.3 (The Nordic Cochrane Centre, Copenhagen, Denmark). A $P$-value $<0.05$ was considered statistically significant, except where otherwise specified.

\section{Results}

\section{Study selection and characteristics}

A total of 348 reports were retrieved from the search of the mentioned two databases, using the described search strategies. After excluding reports that were duplicated or irrelevant to our meta-analysis, 17 studies that assessed IGF-1R expression in patients with BSTSs were considered eligible for inclusion in this study. By full-text review, nine were excluded, as two papers were assessed by other methods, such as polymerase chain reaction (PCR), western blotting, and enzyme-linked immunosorbent assay (ELISA), and survival data was not available from published data or the authors in seven studies. One further study was excluded because the number of patients with negative IGF-1R was only two. Finally, seven studies that met the inclusion criteria were included in this meta-analysis (Figure 1). The seven studies comprised 627 patients (sample ranges from 31 to

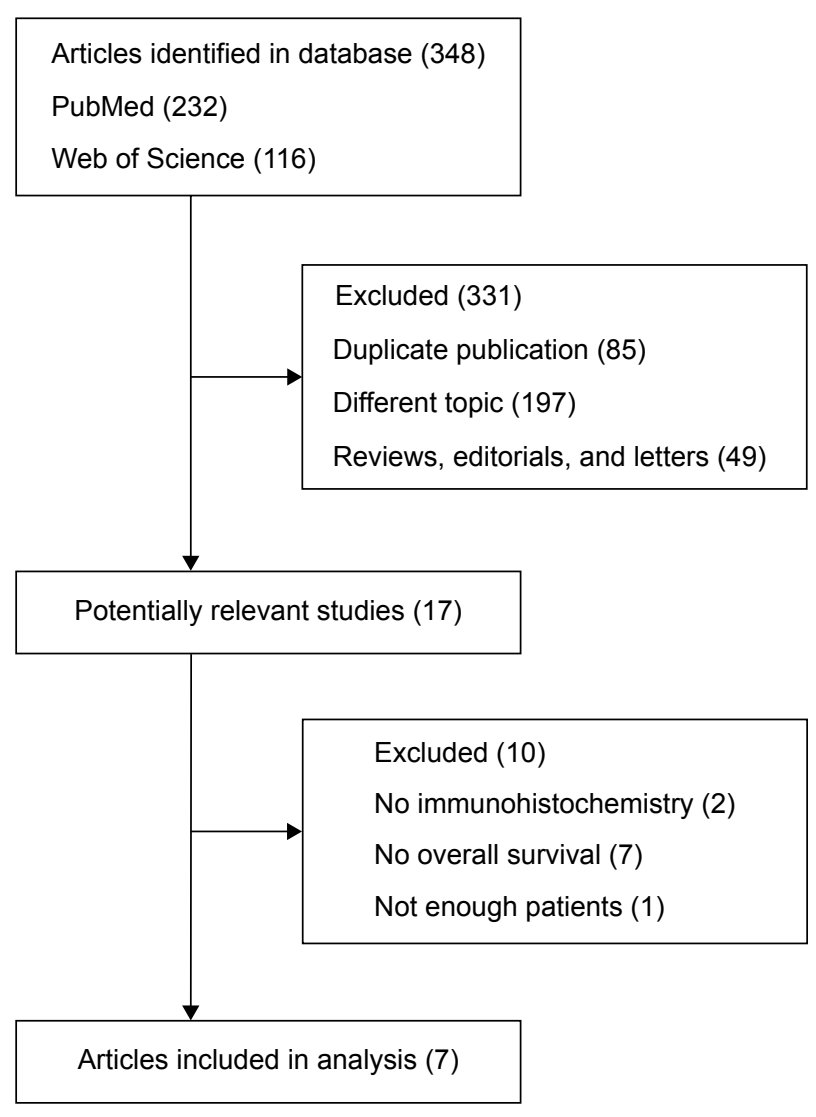

Figure I Flowchart of included studies.
290 patients). Among these seven studies, two were research on osteosarcoma, ${ }^{11,12}$ two were on EWS, ${ }^{15,16}$ and one each were on SS, liposarcoma, and rhabdomyosarcoma (Table 2). ${ }^{17-19}$ Two papers were by the same first author, ${ }^{12,16}$ but different samples and tumor types were focused in these two reports. In five out of seven studies, ${ }^{12,15,16,18,19}$ the cutoff survival was set as staining in $\geq 10 \%$ of cells. In a study by Wang ${ }^{11}$ the cutoff was staining in $\geq 25 \%$ of cells, and in the study by Palmerini, the cutoff was set as positive in staining. ${ }^{17}$

\section{High IGF-IR expression and OVS in BSTSs}

The correlation of IGF-1R expression and OVS of different types of BSTS patients was illustrated in a forest plot of HR with a fixed-effects model (Figure 2). Overall, elevated expression of IGF-1R in BSTSs was significantly associated with mortality risk for patients in the random-effects model (pooled HR $=2.15,95 \%$ CI: $1.06-4.38 ; P=0.03$ ). High heterogeneity was shown in the analysis of sarcomas with IGF-1R expression $\left(I^{2}=88 \%, P<0.001\right)$.

To explore the source of heterogeneity, subgroup analyses were conducted by subtype of sarcomas. As $I^{2}$ of the osteosarcoma subgroup and EWS subgroup was $46 \%$ and $69 \%$, respectively, the fixed-effects model and randomeffects model were utilized to pool the results. Our further analysis indicated that high expression of IGF-1R in osteosarcoma was significantly related to poor prognosis (pooled $\mathrm{HR}=2.20,95 \%$ CI: $1.59-3.03 ; P<0.001)$. However, in EWS, IGF-1R seemed not to play a predictive role of outcome (pooled HR $=1.01,95 \%$ CI: 0.45-2.27; $P=0.99$ ) (Table 3).

The source of patients and sample sizes were also evaluated (Table 3). Six studies were based on Western populations (five from Europe and one from North America), and the remaining one research was based on an Eastern population (from the People's Republic of China). As there was high heterogeneity $\left(I^{2}=81 \%\right)$, the random-effects model was used to perform the analysis. The source of patients was not directly related to the outcome of BSTSs (pooled HR $=2.22,95 \%$ CI: 0.90-5.48; $P=0.08$ ). Concerning sample sizes, IGF-1R expression in small-size studies $(\mathrm{n}<50)$ was considered to be not correlated with outcome (combined HR $=2.18,95 \%$ CI: $0.67-7.07 ; P=0.20$ ), and the elevated expression in large-size studies $(\mathrm{n}>50)$ also revealed no relation with the outcome of BSTS patients (pooled HR $=2.15$, 95\% CI: $0.88-5.28 ; P=0.09$ ).

\section{Publication bias}

Funnel plots were conducted to detect the potential publication bias of the enrolled studies for OVS. The funnel plot is 
Table 2 Characteristics of the seven studies included in the meta-analysis

\begin{tabular}{|c|c|c|c|c|c|c|c|}
\hline Study (author, year) & Patient source & $\begin{array}{l}\text { Number } \\
\text { of patients }\end{array}$ & Tumor type & $\begin{array}{l}\text { Analysis } \\
\text { method }\end{array}$ & Outcome & HR estimation & $\begin{array}{l}\text { NOS } \\
\text { score }\end{array}$ \\
\hline van de Luijtgaarden et al ${ }^{12} 2013^{*}$ & The Netherlands & 44 & Osteosarcoma & $\mathrm{IHC}$ & OVs & $0.76(0.16-3.6 I)^{\mathrm{a}, \mathrm{b}}$ & 7 \\
\hline Wang et al' 2012 & People's Republic of China & 84 & Osteosarcoma & $\mathrm{IHC}$ & OVS & $2.30(1.66-3.20)$ & 8 \\
\hline Scotlandi et al ${ }^{15} 201 \mathrm{I}$ & Italy & 290 & Ewing sarcoma & $\mathrm{IHC}$ & OVs & $0.77(0.63-0.93)^{\mathrm{a}}$ & 8 \\
\hline van de Luijtgaarden et al ${ }^{16} 2013^{* *}$ & The Netherlands & 36 & Ewing sarcoma & $\mathrm{IHC}$ & OVs & $\mathrm{I} .89(0.6 \mathrm{I}-5.86)^{\mathrm{a}, \mathrm{b}}$ & 7 \\
\hline van Gaal et al ${ }^{19} 2013$ & The Netherlands & 54 & Rhabdomyosarcoma & $\mathrm{IHC}$ & OVs & $18.02(2.52-128.86)^{\mathrm{a}}$ & 6 \\
\hline Palmerini et al ${ }^{17} 2015$ & Italy & 88 & Synovial sarcoma & $\mathrm{IHC}$ & OVs & $2.50(\mathrm{I} . \mathrm{II}-5.63)^{\mathrm{a}}$ & 8 \\
\hline Cheng et al ${ }^{18} 2009$ & Canada & 31 & Liposarcoma & $\mathrm{IHC}$ & OVS & $7.44(1.59-34.81)$ & 8 \\
\hline
\end{tabular}

Notes: aData extracted from given information. 'Original data obtained from the author. *Osteosarcoma. **Ewing's sarcoma.

Abbreviations: HR, hazard ratio; IHC, immunohistochemistry; NOS, Newcastle-Ottawa Scale; OVS, overall survival.

presented in Figure 3. The funnel plot shapes showed that the included studies did not exhibit apparent asymmetry. These results indicated the absence of publication bias of our meta-analysis.

\section{Discussion}

Amplification of IGF-1R expression has been shown in many human solid malignancies, including lung cancer, breast cancer, and colorectal cancer, etc, ${ }^{22-25}$ and has been related to poor outcome. In animal studies, increased expression of IGF-1R was revealed to be correlated with worse survival in canine osteosarcoma. ${ }^{26,27}$ Elevated expression of IGF-1R was also found to induce the transformation of fibroblasts and development of tumors in nude mice. ${ }^{28}$ IGF-1R has caught researchers' attention for its potential in targeted therapy. Numerous clinical trials have either been completed or are underway. However, the response rate to therapy with IGF-1R specific agent alone was approximately $10 \%-15 \%$, in patients with relapsed, heavily pretreated sarcomas. ${ }^{4}$ Mechanisms of resistance to IGF-1R inhibition are being studied. ${ }^{29,30}$ Combination therapy has also been evaluated in quite a few studies, but no objective responses were observed in a recently reported Phase II study on anti-IGF-1R antibody in combination with mTOR inhibitor, ${ }^{31}$ despite the promising results in preclinical models and an earlier report on the same combination conducted by another institution, which showed clinical activity, ${ }^{32}$ Researchers have also tried to find the correlation of IGF-1R expression with the sensitivity of sarcomas when treated with IGF-1R monoclonal antibodies (mAbs). A clinical trial indicated that presence of IGF-1R served as a biomarker to predict elevated sensitivity of sarcomas to IGF-1R-targeted therapy. ${ }^{9}$ However, opposite opinions were given in repeated studies that showed IGF-1R expression by IHC was not predictive of clinical outcome after treatment with IGF-1R mAb in combination with mTOR inhibitors. ${ }^{32,33}$ More studies are needed to further analyze the controversial role IGF-1R plays in predicting the outcome of sarcoma patients treated by IGF-1R mAbs. Thus, the prognostic significance of IGF-1R expression in outcome of BSTS patients with conventional therapeutic strategies is meaningful.

To the best of our knowledge, this is the first meta-analysis to focus on the correlation between IGF-1R expression and BSTS patient outcomes. Our research included seven articles and 627 BSTS patients from four countries. We demonstrated that high IGF-1R expression was a marker of aggressive disease, associated with poorer OVS of BSTS patients. Subgroup analysis also showed interesting results. In osteosarcoma, elevated IGF-1R expression was significantly related to worse prognosis according to our study, based on 128

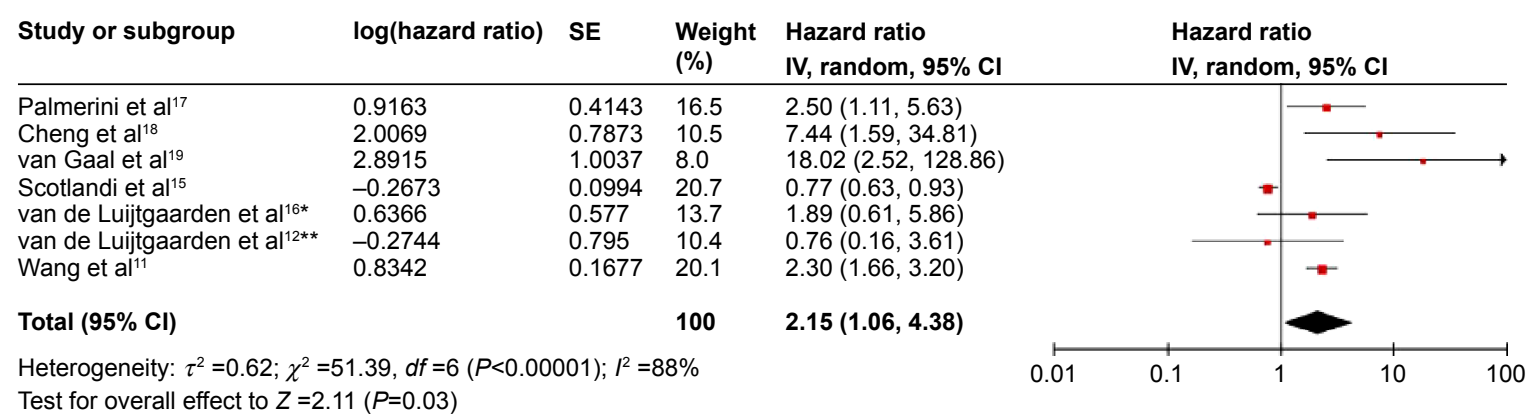

Figure 2 Meta-analysis of impact of IGF-IR expression on overall survival of patients with sarcomas.

Notes: Results are presented as individual and pooled HR and $95 \% \mathrm{Cl}$. *Ewing's sarcoma. **Osteosarcoma.

Abbreviations: $\mathrm{Cl}$, confidence interval; $\mathrm{HR}$, hazard ratio; $\mathrm{SE}$, standard error. 
Table 3 Meta-analysis of overall and subgroup analysis for IGF-IR expression and OVS in BSTSs

\begin{tabular}{|c|c|c|c|c|c|c|c|c|c|}
\hline \multirow[t]{2}{*}{ Categories } & \multirow[t]{2}{*}{ Studies } & \multirow[t]{2}{*}{ Patients } & \multirow[t]{2}{*}{ HR (95\% Cl) } & \multirow[t]{2}{*}{ Model } & \multicolumn{5}{|c|}{ Heterogeneity } \\
\hline & & & & & $x^{2}$ & $12 \%$ & $P$-value & $z$ & $P$-value \\
\hline OVs & 7 & 627 & $2.15(1.06-4.38)$ & Random & 51.39 & 88 & $<0.001$ & 2.11 & 0.03 \\
\hline \multicolumn{10}{|l|}{ Tumor type } \\
\hline Osteosarcoma & 2 & 128 & $2.20(1.59-3.03)$ & Fixed & 1.86 & 46 & 0.17 & 4.80 & $<0.001$ \\
\hline Ewing sarcoma & 2 & 326 & I.0I (0.45-2.27) & Random & 2.38 & 58 & 0.12 & 0.01 & 0.99 \\
\hline \multicolumn{10}{|l|}{ Geography } \\
\hline Western & 6 & 543 & $2.22(0.90-5.48)$ & Random & 26.51 & 81 & $<0.001$ & 1.73 & 0.08 \\
\hline Eastern & 1 & 84 & $2.30(1.66-3.20)$ & Random & - & - & - & - & - \\
\hline \multicolumn{10}{|l|}{ Sample size } \\
\hline Smaller size $(\mathrm{n}<50)$ & 3 & 111 & $2.18(0.67-7.07)$ & Random & 4.25 & 53 & 0.12 & 1.30 & 0.20 \\
\hline Larger size $(n \geq 50)$ & 4 & 516 & $2.15(0.88-5.28)$ & Random & 44.35 & 93 & $<0.001$ & 1.68 & 0.09 \\
\hline
\end{tabular}

Abbreviations: BSTS, bone and soft tissue sarcoma; $\mathrm{Cl}$, confidence interval; $\mathrm{HR}$, hazard ratio; OVS, overall survival; -, no data.

samples. Nevertheless, IGF-1R expression had a slight trend to have a favorable role in EWS patients (326 samples), albeit with no statistical significance. To further analyze this, we tried to include a paper by Mora et $\mathrm{al}^{34}$ on EWS in our additional analysis, which was initially excluded as there were only two subject in IGF-1R-negative group. Surprisingly, we discovered that there was statistical significance between IGF-1R expression and favorable outcome of EWS patients (data not shown), but we have to admit that more data are needed to confirm the favorable role of IGF-1R expression in EWS. No correlation was found between outcomes and sample sizes or geographical distribution of patient.

Although our study is the first meta-analysis on the relation between IGF-1R expression and clinical outcomes of BSTS patients, several limitations still exist. BSTSs are rare subgroups of malignancies, and most IGF-1R-related studies have involved patients treated with IGF-1R inhibitors or $\mathrm{mAbs}$, which will obviously alter the expression of IGF-1R and IGF-1R-related survival. There was only one study each for SS, liposarcoma, and rhabdomyosarcoma included in our

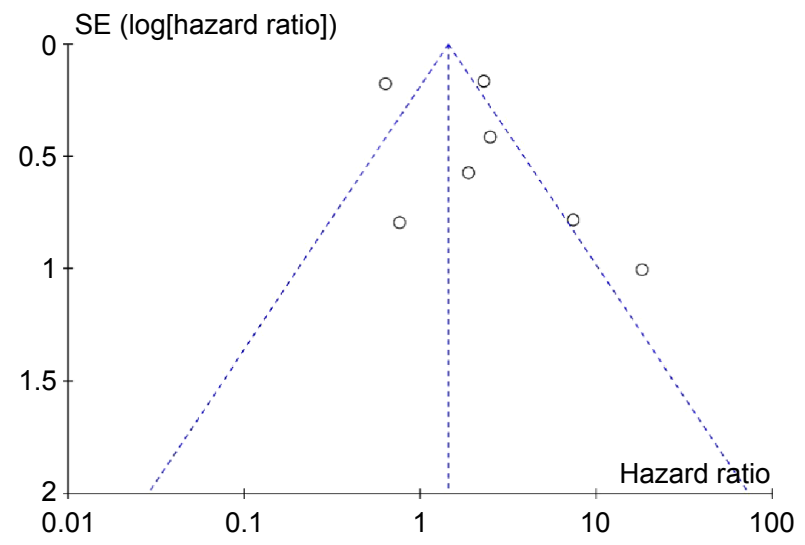

Figure 3 Funnel plot for the evaluation of potential publication bias in the impact of IGF-IR expression on overall survival of patients with sarcomas. report, as a result of fewer incidences. More data for these three types of BSTSs are needed. Further, we extracted data from survival curves in several papers where detailed data, such as HR and $95 \% \mathrm{CI}$, were not presented directly. The calculated HRs and $95 \%$ CIs were possibly not as reliable as given data. Furthermore, publication bias cannot be totally ruled out because negative studies are not as acceptable as positive results. Subgroup analysis for OVS was conducted according to cancer subtype, source of patients, and sample size. The subgroup analysis might explain a part of the heterogeneity.

In conclusion, our meta-analysis demonstrated that elevated IGF-1R expression was significantly associated with adverse clinical outcome. Our study revealed that IGF-1R might be an independent biomarker for BSTS patients, especially patients suffering from osteosarcoma. To strengthen our conclusion, more standardized prospective studies with high quality are still needed to evaluate the prognostic value of IGF-1R.

\section{Acknowledgment}

This work was supported by National Natural Science Foundation of the People's Republic of China (grant numbers 81172547 and 30973444).

\section{Disclosure}

The authors report no conflicts of interest in this work.

\section{References}

1. Biermann JS, Adkins DR, Benjamin RS, et al. Bone cancer. J Natl Compr Canc Netw. 2010;8(6):688-712.

2. Sun L, Li Y, Zhang J, Li H, Li B, Ye Z. Prognostic value of pathologic fracture in patients with high grade localized osteosarcoma: a systemic review and meta-analysis of cohort studies. J Orthop Res. 2015;33(1):131-139.

3. McKinsey EL, Parrish JK, Irwin AE, et al. A novel oncogenic mechanism in Ewing sarcoma involving IGF pathway targeting by EWS/ Fli1-regulated microRNAs. Oncogene. 2011;30(49):4910-4920. 
4. Borinstein SC, Barkauskas DA, Krailo M, et al. Investigation of the insulinlike growth factor-1 signaling pathway in localized Ewing sarcoma: a report from the Children's Oncology Group. Cancer. 2011;117(21):4966-4976.

5. Pappo AS, Vassal G, Crowley JJ, et al. A phase 2 trial of R1507, a monoclonal antibody to the insulin-like growth factor-1 receptor (IGF-1R), in patients with recurrent or refractory rhabdomyosarcoma, osteosarcoma, synovial sarcoma, and other soft tissue sarcomas: results of a Sarcoma Alliance for Research Through Collaboration study. Cancer. 2014;120(16): 2448-2456.

6. Chugh R, Griffith KA, Davis EJ, et al. Doxorubicin plus the IGF-1R antibody cixutumumab in soft tissue sarcoma: a phase I study using the TITE-CRM model. Ann Oncol. 2015;26(7):1459-1464.

7. Scotlandi K, Picci P. Targeting insulin-like growth factor 1 receptor in sarcomas. Curr Opin Oncol. 2008;20(4):419-427.

8. Rikhof B, de Jong S, Suurmeijer AJ, Meijer C, van der Graaf WT. The insulin-like growth factor system and sarcomas. J Pathol. 2009;217(4): 469-482.

9. Asmane I, Watkin E, Alberti L, et al. Insulin-like growth factor type 1 receptor (IGF-1R) exclusive nuclear staining: a predictive biomarker for IGF-1Rmonoclonal antibody (Ab) therapy in sarcomas. Eur JCancer. 2012; 48(16):3027-3035

10. Xie Y, Skytting B, Nilsson G, Brodin B, Larsson O. Expression of insulin-like growth factor-1 receptor in synovial sarcoma: association with an aggressive phenotype. Cancer Res. 1999;59(15):3588-3591.

11. Wang YH, Han XD, Qiu Y, et al. Increased expression of insulin-like growth factor-1 receptor is correlated with tumor metastasis and prognosis in patients with osteosarcoma. J Surg Oncol. 2012;105(3):235-243.

12. van de Luijtgaarden AC, Roeffen MH, Leus MA, et al. IGF signaling pathway analysis of osteosarcomas reveals the prognostic value of pAKT localization. Future Oncol. 2013;9(11):1733-1740.

13. Wang Y, Lipari P, Wang X, et al. A fully human insulin-like growth factor-I receptor antibody SCH 717454 (Robatumumab) has antitumor activity as a single agent and in combination with cytotoxics in pediatric tumor xenografts. Mol Cancer Ther. 2010;9(2):410-418.

14. Duan Z, Choy E, Harmon D, et al. Insulin-like growth factor-I receptor tyrosine kinase inhibitor cyclolignan picropodophyllin inhibits proliferation and induces apoptosis in multidrug resistant osteosarcoma cell lines. Mol Cancer Ther. 2009;8(8):2122-2130.

15. Scotlandi K, Manara MC, Serra M, et al. Expression of insulin-like growth factor system components in Ewing's sarcoma and their association with survival. Eur J Cancer. 2011;47(8):1258-1266.

16. van de Luijtgaarden AC, Versleijen-Jonkers YM, Roeffen $\mathrm{MH}$, Schreuder HW, Flucke UE, van der Graaf WT. Prognostic and therapeutic relevance of the IGF pathway in Ewing's sarcoma patients. Target Oncol. 2013;8(4):253-260.

17. Palmerini E, Benassi MS, Quattrini I, et al. Prognostic and predictive role of CXCR4, IGF-1R and Ezrin expression in localized synovial sarcoma: is chemotaxis important to tumor response? Orphanet J Rare Dis 2015;10:6

18. Cheng H, Dodge J, Mehl E, et al. Validation of immature adipogenic status and identification of prognostic biomarkers in myxoid liposarcoma using tissue microarrays. Hum Pathol. 2009;40(9):1244-1251.
19. van Gaal JC, Roeffen MH, Flucke UE, et al. Simultaneous targeting of insulin-like growth factor-1 receptor and anaplastic lymphoma kinase in embryonal and alveolar rhabdomyosarcoma: a rational choice. Eur J Cancer. 2013;49(16):3462-3470.

20. Ahlén J, Wejde J, Brosjö O, et al. Insulin-like growth factor type 1 receptor expression correlates to good prognosis in highly malignant soft tissue sarcoma. Clin Cancer Res. 2005;11(1):206-216.

21. Tierney JF, Stewart LA, Ghersi D, Burdett S, Sydes MR. Practical methods for incorporating summary time-to-event data into meta-analysis. Trials. 2007;8:16.

22. Kim WY, Jin Q, Oh SH, et al. Elevated epithelial insulin-like growth factor expression is a risk factor for lung cancer development. Cancer Res. 2009;69(18):7439-7448.

23. Sheen-Chen SM, Chou FF, Hsu W, Huang CC, Eng HL, Tang RP. Lack of prognostic value of insulin-like growth factor-1 in patients with breast cancer: analysis with tissue microarray. Anticancer Res. 2007;27(5B): 3541-3544.

24. Koda M, Reszec J, Sulkowska M, Kanczuga-Koda L, Sulkowski S. Expression of the insulin-like growth factor-I receptor and proapoptotic Bax and Bak proteins in human colorectal cancer. Ann N Y Acad Sci. 2004;1030: 377-383.

25. Heidegger I, Kern J, Ofer P, Klocker H, Massoner P. Oncogenic functions of IGF1R and INSR in prostate cancer include enhanced tumor growth, cell migration and angiogenesis. Oncotarget. 2014;5(9):2723-2735.

26. Maniscalco L, Iussich S, Morello E, et al. Increased expression of insulin-like growth factor-1 receptor is correlated with worse survival in canine appendicular osteosarcoma. Vet J. Epub 2014 Sep 10.

27. MacEwen EG, Pastor J, Kutzke J, et al. IGF-1 receptor contributes to the malignant phenotype in human and canine osteosarcoma. J Cell Biochem. 2004;92(1):77-91.

28. Kaleko M, Rutter WJ, Miller AD. Overexpression of the human insulinlike growth factor I receptor promotes ligand-dependent neoplastic transformation. Mol Cell Biol. 1990;10(2):464-473.

29. Fahrenholtz CD, Greene AM, Beltran PJ, Burnstein KL. A novel calciumdependent mechanism of acquired resistance to IGF-1 receptor inhibition in prostate cancer cells. Oncotarget. 2014;5(19):9007-9021.

30. Kang Z, Yu Y, Zhu YJ, et al. Downregulation of IGFBP2 is associated with resistance to IGF1R therapy in rhabdomyosarcoma. Oncogene. 2014; 33(50):5697-5705.

31. Wagner LM, Fouladi M, Ahmed A, et al. Phase II study of cixutumumab in combination with temsirolimus in pediatric patients and young adults with recurrent or refractory sarcoma: a report from the Children's Oncology Group. Pediatr Blood Cancer. 2015;62(3):440-444.

32. Schwartz GK, Tap WD, Qin LX, et al. Cixutumumab and temsirolimus for patients with bone and soft-tissue sarcoma: a multicentre, open-label, phase 2 trial. Lancet Oncol. 2013;14(4):371-382.

33. Cao Y, Roth M, Piperdi S, et al. Insulin-like growth factor 1 receptor and response to anti-IGF1R antibody therapy in osteosarcoma. PLoS One. 2014;9(8):e106249.

34. Mora J, Rodríguez E, de Torres C, et al. Activated growth signaling pathway expression in Ewing sarcoma and clinical outcome. Pediatr Blood Cancer. 2012;58(4):532-538.
OncoTargets and Therapy

\section{Publish your work in this journal}

OncoTargets and Therapy is an international, peer-reviewed, open access journal focusing on the pathological basis of all cancers, potential targets for therapy and treatment protocols employed to improve the management of cancer patients. The journal also focuses on the impact of management programs and new therapeutic agents and protocols on

\section{Dovepress}

patient perspectives such as quality of life, adherence and satisfaction. The manuscript management system is completely online and includes a very quick and fair peer-review system, which is all easy to use. Visit http://www.dovepress.com/testimonials.php to read real quotes from published authors. 